\title{
Anagram solution as a function of pronounceability and difficulty
}

JOHN A. HEBERT AND CECIL A. ROGERS, JR. UNIVERSITY OF ARIZONA

The present study investigates the effect of pronounceability (P), as rated by $S$ s, on anagram solution probability under two levels of difficulty (D), or number of letters moved. From 60 five-letter anagrams rated for $P$, four lists of 10 anagrams each were constructed to represent both easy-to-pronounce and hard-to-pronounce anagrams in both levels of $D$ (one vs. two letters moved). The results indicate that $\mathrm{P}$ is inversely related to solution probability, and that $D$ is not an effective variable with hard-to-pronounce anagrams.

Ekstrand \& Dominowski (1965), supporting the Beilin \& Horn (1962) study, have demonstrated that non-word anagrams are less difficult to solve for other words (e.g., teirw for write) than word anagrams for other words (e.g., melon for lemon). The former investigators suggest that representational responses necessary for the perception of the stimulus, as well as implicit associative responses, interfere with solution. The latter investigators suggest that $\mathrm{S}$ perseverates on the sound or meaning of the word anagrams. Recently, however, Mayzner \& Tresselt (1965) presented evidence that there is no difference in solution times of word and non-word anagrams. This discrepancy of results suggests that perhaps an alternate approach is needed to elucidate the problem of word vs. non-word anagrams.

It is reasonable to consider words more pronounceable than non-words. It is equally reasonable to consider some non-words more pronounceable than other nonwords. In light of the Beilin \& Horn(1962) and Ekstrand \& Dominowski (1965) findings, it is suggested that pronounceability (P) may interfere with word anagram solution. If this is the case, it follows that $P$ should have a similar, though perhaps reduced, effect on the solution of non-word anagrams. For example, if one takes two anagrams of equal letter order difficulty and Thorndike-Lorge frequency, e.g., crove (solutioncover) and mcusi (solution-music), Ss should rate crove higher in $\mathrm{P}$ than mcusi. Differences in solution probabilities may then be attributable to $P$. This study investigates the effect of $P$, as rated by Ss, upon the probability of solution of non-word anagrams under two levels of difficulty (D), or number of letters moved.

Method

Materials. Thirty Thorndike-Lorge AA nouns were randomly chosen as potential solution words from a list of five-letter nouns beginning with consonants. Sixty anagrams (30 one-letter move and 30 two-letter move problems) were then constructed by $\mathrm{E}$ so as to lend themselves easily to division into high pronounceability (HP) and low pronounceability (LP) categories.
Two hundred and ten Ss then rated the 60 anagrams on a 1 to 5 scale for $\mathrm{P}$ (low to high). Each $\mathrm{S}$ rated a list of 10 anagrams presented in a random sequence or its inverse. All lists contained expected HP and LP problems. Each anagram was rated by 35 Ss. This procedure yielded four types of anagrams: (1) HP oneletter move or "easy" anagrams (HP-E), (2) HP twoletter move or "hard" anagrams (HP-H), (3) LP oneletter move anagrams (LP-E), and (4) LP two-letter move anagrams (LP-H). The 10 items having the smallest variance in each of the four categories of anagrams were then selected for one of four lists, since no overlap in ratings of the expected HP and LP items occurred. The HP-E list, (e.g., patin, crove, and larbo with solutions-paint, cover, and labor) had a mean P rating of 4.66 , and a range of 4.91 to 4.34 , while in the $\mathrm{HP}-\mathrm{H}$ list (e.g., rocut, sumic, and doblo with solutions-court, music, and blood) the mean $=4.59$, and range $=4.94$ to 4.29. In the LP-E list (e.g., sgaru, mcusi, and trnai with solutions-sugar, music, and train) the mean $=2.29$, and range $=2.63$ to 1.86 , while in the LP-H list (e.g., drkni, if $g$ th, and mnoye with solutions-drink, fight, and money) mean $=1.98$, range $=2.57$ to 1.45 . Some of the solutions, e.g., sugar, were used in more than one list. Note that bigram frequency (Mayzner \& Tresselt, 1959) was not controlled in the lists. Since experimental control was found to be impractical, statistical control of bigram frequency was then used.

The 10 items for the four lists were placed on an $8-1 / 2$ in. $x 5-1 / 2$ in. verbal booklet so that only one item could be seen or worked on at a time. The booklet consisted of 12 pages: a cover page, an instruction page, and 10 problem pages. One problem was dittoed on the center of each page, with all of the letters capitalized and one typewriter space apart.

Subjects and procedure. Employing a 2 by 2 factorial design, 240 additional $\mathrm{Ss}$ were assigned to the test session. All of the Ss in the rating and testing sessions were introductory psychology students. Sixty Ss were randomly assigned to a list, with six Ss assigned to each of 10 orders of a given list. Upon receiving general instructions, Ss turned to the instruction page where they were told what an anagram was and what the task was. E then paced the group of $260 \mathrm{Ss}$ through the 10 items, allowing 13 sec. per problem and a 2 sec. inter-item interval.

\section{Results and Discussion}

The analysis of covariance technique was used to control for bigram frequency, as calculated by the Underwood \& Schulz (1960) counts. Bigram frequency of a given 
anagram was treated as a covariate with the total number of solutions for that anagram. After correction for the effects of bigram frequency, $\mathrm{P}$ was found to be significant at the .001 level $(F=28.06, d f=1 / 35)$ whereas $D$ was not significant $(F=0.16, \mathrm{df}=1 / 35)$. The $\mathrm{P}$ by $\mathrm{D}$ interaction, however, was significant at the .01 level $(\mathrm{F}=8.10, \mathrm{df}=1 / 35)$. As expected, the LP-E and LP-H lists yielded higher mean number of solutions (6.90 and 4.24 respectively) than did the HP-E and HP-H lists ( 3.03 and 3.31 respectively). Note that these values for means are corrected means based on the analysis of covariance (Fig, 1). Since LP-E and HP-E lists were expected to be less difficult than their counterpart $\mathrm{H}$ lists, a slight reversal occurred with the HP-E and HP-H list means. Orthogonal comparisons, however, indicate that no significant difference exists between the HP-E and HP-H means.

These results demonstrate that $P$ is inversely related to solution probability, which lends support to the Beilin

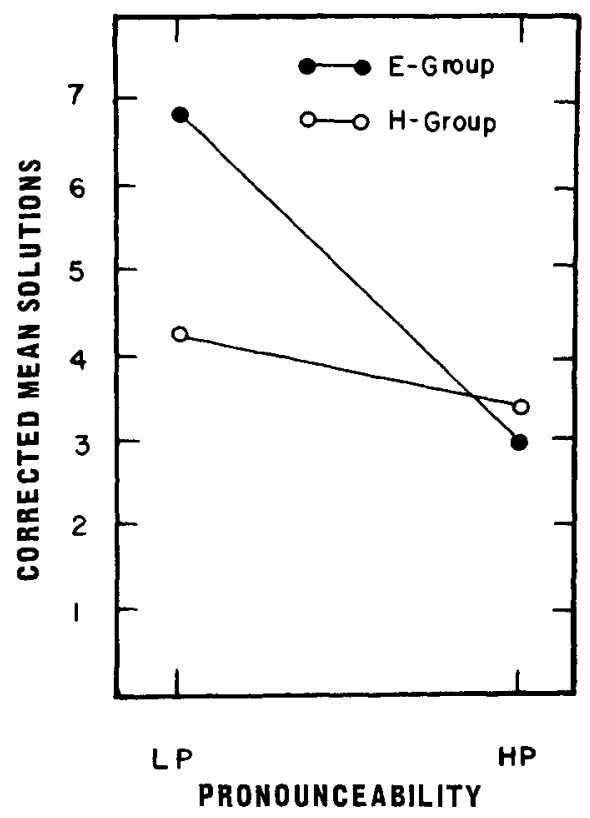

Fig. 1. Corrected mean number of solutions for $E$ and $H$ groups as a function of $P$.
\& Horn (1962) and Ekstrand \& Dominowski (1965) findings. Further, since the HP-E and HP-H groups appear to be essentially equal, the $P$ by $D$ interaction is interpreted to mean that $D$ is an effective variable only in the absence of the HP condition. It could be that $P$ is so effective in the HP condition that the effects of $D$ are nullified.

The finding of greatest importance is the inverse relationship between $P$ and solution probability. It seems quite likely that HP anagrams are more readily encoded than LP anagrams. It is reasonable to consider HP items more "collapsible" into an encodable unit than LP problems, by virtue of $P$. When confronted with an anagram, $S$ attempts to encode the stimulus as a unit because of prior reading habits. In order to accomplish this with LP anagrams, S may immediately have to rearrange the combination of letters to perceive an encodable unit, whereas the HP problem is encodable as is. The immediate rearranging of LP anagrams increases the probability of solution relative to HP items.

The discrepancy between the Beilin \& Horn (1962) and Ekstrand \& Dominowski (1965) studies vs. the Mayzner \& Tresselt (1965) finding perhaps is attributable to effects of $\mathbf{P}$. According to the above hypothesis, a sample of non-word anagrams equal in $\mathrm{P}$ to a sample of word anagrams should have equal solution probabilities. Therefore, the problem of word vs. non-word anagrams is meaningful only in terms of $P$ under this hypothesis. It should be noted, however, that associations to a stimulus could also interfere with solution as Mayzner \& Tresselt (1965) and Ekstrand \& Dominowski (1965) have both suggested. Such interference, however, may serve to augment the effect of $\mathbf{P}$ since the most readily encoded problems will most likely be first to produce associations.

\section{References}

Beilin, H., \& Hom, R. Transition probability effects in anagram problem solving. J. exp. Psychol., 1962, 63, 514-518.

Ekstrand, B. R., \& Dominowski, R. L. Solving words as anagrams. Psychon. Sci., 1965, 2, 239-240.

Mayzner, M. S., \& Tresselt, M. E. Anagram solution times: A function of transition probabilities. J. Psychol., 1959, 47, 117-125.

Mayzner, M. S., \& Tresselt, M. E. Solving words as anagrams: An issue re-examined. Psychon. Sci., 1965, 3, 363-364.

Underwood, B. J, \& Schulz, R. W. Meaningfulness and verbal learning. Chicago: J. P. Lippincott Company, 1960. 